\title{
Ratio of Tiling Generating Functions of semi-Hexagons and Quartered Hexagons with Dents
}

\author{
Tri Lai* \\ Department of Mathematics, University of Nebraska - Lincoln, Lincoln, NE 68588, U.S.A. \\ Email: tlai3@unl.edu
}

Received: July 1, 2021, Accepted: August 17, 2021, Published: August 27, 2021

The authors: Released under the CC BY-ND license (International 4.0)

ABSTRACT: We consider the tiling generating functions of semi-hexagons and quartered hexagons with dents on their sides. In general, there are no simple product formulas for these generating functions. However, we show that modifying the regions' width changes the tiling generating functions by only a simple multiplicative factor.

Keywords: Lozenge tilings; Perfect matchings; Plane partitions; Shuffling phenomenon

2020 Mathematics Subject Classification: 05A15; 05B45

\section{Introduction}

In general, even a minor modification of a region would lead to an unpredictable change in its tiling number. However, in some situations, it changes the tiling number by only a simple multiplicative factor. The author and Rohatgi first introduced this phenomenon for the doubly-dented hexagons in [21] and named it the "Shuffling Phenomenon" ${ }^{\dagger}$ for tilings.

The initial example of this phenomenon was recognized earlier when the author attended the Joint Mathematics Meetings 2018. After discussing with Dennis Stanton about the tiling number of Ciucu and Krattenthaler's 'S-cored hexagon' (a hexagon with a cluster of four triangles removed) [5], the author found a striking pattern in the tiling number of the region when the side-lengths of the $S$-core are changed. This example was later generalized in $[6,18,19]$. The phenomenon has been found in many different forms and different region families. For recent work about the phenomenon, we refer the reader to, e.g. $[3,4,8,12,13,17,20]$.

In this paper, we show several new instances of the shuffling phenomenon. In particular, we are investigating two new region families. The first family is a class of semi-hexagons, i.e., upper halves of symmetric hexagons (see Figure 1). The second family consists of certain quartered hexagons, i.e., halves of symmetric semi-hexagons (see Figure 4). We show that the tiling generating functions of these regions change by only a simple multiplicative factor if we adjust the width while fixing the other parameters. A highlight of the result is that these tiling generating functions are not given by simple product formulas themselves.

We want to emphasize that most of the results in the field of enumeration of tilings are unweighted enumerations; the weighted enumerations are very rare. This paper is devoted to such rare enumerations. The unweighted version of Theorem 1.1 was independently found by Condon [8]. Strictly speaking, Condon investigates a different family of regions, namely hexagons with dents on two non-adjacent sides. However, his regions have the same tiling number as our regions.

We now define in detail the semi-hexagon with dents on two sides. Consider a trapezoidal region of sidelengths $x, m+n, x+m+n, m+n$ (in clockwise order, starting from the top ${ }^{\ddagger}$ ) in the triangular lattice, as shown in Figure 1(a). Next, we remove $m$ up-pointing unit triangles from the left side and $n$ up-pointing unit triangles from the right side. These removed triangles are called the 'dents' and are indicated by the black triangles in the figure. A lozenge is a union of any two unit triangles that share an edge (see Figure 2 for three possible orientations of the lozenges: vertical, left, and right). A lozenge tiling of a region is a covering of the region by

\footnotetext{
*This research was supported in part by Simons Foundation Collaboration Grant (\# 585923)

$\dagger$ The "shuffling phenomenon" here is not related to the "domino shuffling" operation in [10,11].

†Fom now on, we always list the side-lengths of a region in this order.
} 


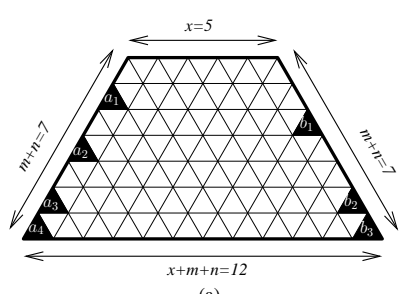

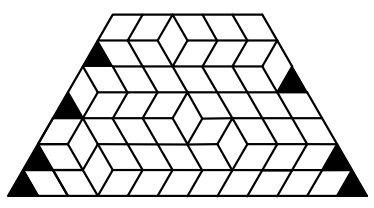

(b)

Figure 1: (a) A semi-hexagon with dents on two sides, and (b) a tiling of its. The black triangles indicate the unit triangles removed.

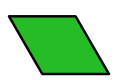

left

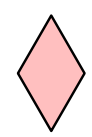

vertical

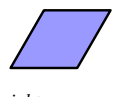

right

Figure 2: Three possible orientations of the lozenges: left, vertical, and right.

lozenges with no gaps or overlaps. See Figure 1(b) for a tiling of a semi-hexagon with dents. In general, our dented semi-hexagon may not have any tiling (see Lemma 2.1 for the tile-ability of this region). Even when it has tilings, the number of tilings is not given by a nice product formula.

We now consider a weight assignment for the lozenges as follows. We define a rectangular coordinate system with the horizontal $i$-axis running along the base of the semi-hexagon; the vertical $j$-axis passes the middle point of the base. A unit on the $i$-axis is equal to $1 / 2$ times the side-length of a lozenge, and a unit on the $j$-axis is equal to $\sqrt{3} / 2$ times the side-length of a lozenge. The vertical lozenge with center at the point $(i, j)$ is weighted by $\frac{X q^{i}+Y q^{-i}}{2}$, where $X, Y, q$ are three indeterminates. The lozenges of different orientations (left and right) are all weighted by 1 . We note that our lozenge-weights are independent of the $j$-ordinate. See Figure 3(a) for an example. The weight of a tilling is now the product of its lozenge-weights ${ }^{\S}$. This weight assignment is a special case of the elliptic weight considered by Borodin, Gorin, and Rains in [2]. Denote by $S_{x}(\mathbf{a}, \mathbf{b})=S_{x}\left(\left(a_{i}\right)_{i=1}^{m} ;\left(b_{j}\right)_{j=1}^{n}\right)$ the resulting weighted region, where $\mathbf{a}=\left(a_{i}\right)_{i=1}^{m}$ and $\mathbf{b}=\left(b_{j}\right)_{j=1}^{n}$ are respectively the sequences of the left dents' positions and the right dents' positions (as they appear from top to bottom).

All regions considered in this paper are weighted regions. Strictly speaking, a 'weighted region' is a pair $(R, \mathrm{wt})$, where $R$ is an unweighted region in the triangular lattice, called the "shape" of the region, and wt is a weight assignment for the tilings of $R$. We will see in the next part of the paper that there exist different weighted regions that have the same shape. We abuse the notation when the weight assignment is clearly given by viewing $R$ as the weighted region. In the rest of the paper, we use the notation $\mathrm{T}(R)$ for the weighted sum of all tilings of $R$. If $R$ does not have any tiling, then $\mathrm{T}(R)=0$. When $R$ is a degenerated region (i.e., a region with an empty interior), we set $\mathrm{T}(R)=1$ by convention. We call $\mathrm{T}(R)$ the tiling generating function of $R$.

In general, the tiling generating function of the semi-hexagon $S_{x}(\mathbf{a}, \mathbf{b})$ is not given by a simple product formula. However, if we consider the ratio of the tiling generating functions of $S_{x}(\mathbf{a}, \mathbf{b})$ and its 'sibling' $S_{y}(\mathbf{a}, \mathbf{b})$, then a magical cancelation happens. (Intuitively, $S_{y}(\mathbf{a}, \mathbf{b})$ is obtained by horizontal stretching or compressing $S_{x}(\mathbf{a}, \mathbf{b})$.) The ratio reduces to a nice product formula.

We often use the standard $q$-Pochhammer symbol in our tiling formulas:

$$
(x ; q)_{n}:= \begin{cases}1 & \text { if } n=0 \\ (1-x)(1-x q) \cdots\left(1-x q^{n-1}\right) & \text { if } n>0 \\ \frac{1}{\left(1-x q^{-1}\right)\left(1-x q^{-2}\right) \cdots\left(1-x q^{n}\right)} & \text { if } n<0 .\end{cases}
$$

We are now ready to state our first main theorem.

Theorem 1.1. Assume that $x, m, n$ are non-negative integers, and $\left(a_{i}\right)_{i=1}^{m}$ and $\left(b_{j}\right)_{j=1}^{n}$ are two increasing sequences of positive integers between 1 and $m+n$. The region $S_{x}\left(\left(a_{i}\right)_{i=1}^{m} ;\left(b_{j}\right)_{j=1}^{n}\right)$ is tile-able if and only if the region $S_{y}\left(\left(a_{i}\right)_{i=1}^{m} ;\left(b_{j}\right)_{j=1}^{n}\right)$ is tile-able. Moreover, if $S_{x}\left(\left(a_{i}\right)_{i=1}^{m} ;\left(b_{j}\right)_{j=1}^{n}\right)$ is tile-able, then we always have

$$
\begin{aligned}
\frac{\mathrm{T}\left(S_{x}\left(\left(a_{i}\right)_{i=1}^{m} ;\left(b_{j}\right)_{j=1}^{n}\right)\right)}{\mathrm{T}\left(S_{y}\left(\left(a_{i}\right)_{i=1}^{m} ;\left(b_{j}\right)_{j=1}^{n}\right)\right)} & =q^{(y-x)\left(\sum_{i=1}^{m} a_{i}+\sum_{j=1}^{n} b_{j}-\frac{(m+n)(m+n+1)}{2}\right)} \frac{\mathrm{PP}_{q^{2}}(y, m, n)}{\mathrm{PP}_{q^{2}}(x, m, n)} \\
& \times \prod_{i=1}^{m} \frac{\left(q^{2(x+i)} ; q^{2}\right)_{a_{i}-i}}{\left(q^{2(y+i)} ; q^{2}\right)_{a_{i}-i}} \prod_{j=1}^{n} \frac{\left(q^{2(x+j)} ; q^{2}\right)_{b_{j}-j}}{\left(q^{2(y+j)} ; q^{2}\right)_{b_{j}-j}}
\end{aligned}
$$

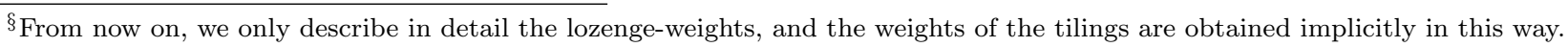



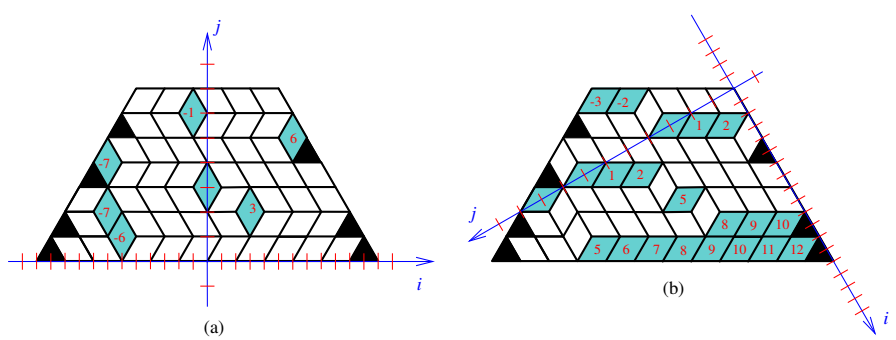

Figure 3: Two weight assignments for the lozenges of a semi-hexagon with dents on two sides. The shaded lozenges passed by the $j$-axis are weighted by $\frac{X+Y}{2}$, the ones with label $k$ are weighted by $\frac{X q^{k}+Y q^{-k}}{2}$.

where

$$
\mathrm{PP}_{q}(a, b, c)=\prod_{i=1}^{a} \prod_{j=1}^{b} \prod_{k=1}^{c} \frac{q^{i+j+k-1}-1}{q^{i+j+k-2}-1}
$$

We note that the ratio of generating functions in (2) does not depend on the determinates $X$ and $Y$ in our weight assignment. We also note that the product $\operatorname{PP}_{q}(a, b, c)$ in the above formula is exactly MacMahon's generating function of the plane partitions fitting in an $(a \times b \times c)$-box [22]. We still do not have any good explanation for the appearance of MacMahon's formula here.

We now re-assign weights to the right lozenges of the semi-hexagon as in Figure 3(b). In particular, the right lozenge with center at $(i, j)$ is weighted by $\frac{X q^{i}+Y q^{-i}}{2}$; all the left and vertical lozenges now have weight 1. Denote by $S_{x}^{\prime}\left(\left(a_{i}\right)_{i=1}^{m} ;\left(b_{j}\right)_{j=1}^{n}\right)$ the new weighted region. In other words, $S_{x}(\mathbf{a}, \mathbf{b})$ and $S_{x}^{\prime}(\mathbf{a}, \mathbf{b})$ have the same shape but different weight assignments. The ratio of the tiling generating functions of $S_{x}^{\prime}(\mathbf{a}, \mathbf{b})$ and $S_{y}^{\prime}(\mathbf{a}, \mathbf{b})$ is also given by a simple product formula. It is, in fact, very similar to that in the previous theorem.

Theorem 1.2. Assume that $x, m, n$ are non-negative integers, and $\left(a_{i}\right)_{i=1}^{m}$ and $\left(b_{j}\right)_{j=1}^{n}$ are two increasing sequences of positive integers between 1 and $m+n$. The region $S_{x}^{\prime}\left(\left(a_{i}\right)_{i=1}^{m} ;\left(b_{j}\right)_{j=1}^{n}\right)$ is tile-able if and only if the region $S_{y}^{\prime}\left(\left(a_{i}\right)_{i=1}^{m} ;\left(b_{j}\right)_{j=1}^{n}\right)$ is tile-able. Moreover, if $S_{x}^{\prime}\left(\left(a_{i}\right)_{i=1}^{m} ;\left(b_{j}\right)_{j=1}^{n}\right)$ is tile-able, then

$$
\begin{aligned}
\frac{\mathrm{T}\left(S_{x}^{\prime}\left(\left(a_{i}\right)_{i=1}^{m} ;\left(b_{j}\right)_{j=1}^{n}\right)\right)}{\mathrm{T}\left(S_{y}^{\prime}\left(\left(a_{i}\right)_{i=1}^{m} ;\left(b_{j}\right)_{j=1}^{n}\right)\right)} & =q^{\frac{n}{2}\left(y^{2}-x^{2}\right)+(y-x)\left(\sum_{i=1}^{n} b_{j}-\frac{1}{2} m^{2}-\frac{1}{2} n^{2}-m n+2 n\right)} \frac{\operatorname{PP}_{q^{2}}(y, m, n)}{\operatorname{PP}_{q^{2}}(x, m, n)} \\
& \times \prod_{j=1}^{n} \prod_{i=1}^{y-x}\left(X^{2}+q^{2\left(x+i-b_{j}\right)} X Y\right) \prod_{i=1}^{m} \frac{\left(q^{2(x+i)} ; q^{2}\right)_{a_{i}-i}}{\left(q^{2(y+i)} ; q^{2}\right)_{a_{i}-i}} \prod_{j=1}^{n} \frac{\left(q^{2(x+j)} ; q^{2}\right)_{b_{j}-j}}{\left(q^{2(y+j)} ; q^{2}\right)_{b_{j}-j}}
\end{aligned}
$$

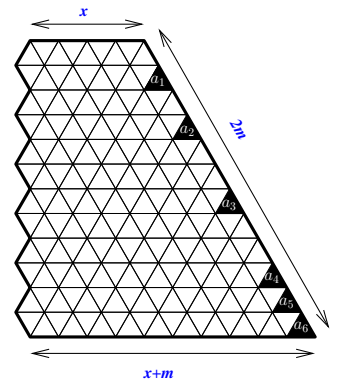

(a)

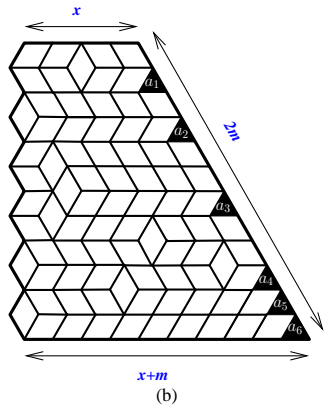

(b)

Figure 4: (a) A quartered with dents on the right side and (b) a tiling of its. The black triangles indicate the unit triangles removed.

Next, we consider a new family of regions called "quartered hexagons" with dents on the right side, as shown in Figure 4. In particular, we consider a right trapezoidal region of side-lengths $x, 2 m, x+m, 2 m$. The vertical left side runs along a zigzag path with $2 m$ steps. We remove $m$ up-pointing unit triangles from the right side of the region at the positions $a_{1}, a_{2}, \ldots, a_{m}$, from top to bottom. We also assign the weights to vertical lozenges of the region, as in Figure 5(a). In particular, the $j$-axis is touching the right side of the region, and the $i$-axis runs along the base. The vertical lozenge with the center at the point $(i, j)$ is weighted by $\frac{q^{i}+q^{-i}}{2}$ (i.e., we set $X=Y=1$ here). Denote by $Q_{x}\left(\left(a_{i}\right)_{i=1}^{m}\right)$ this weighted region. Similar to the case of Theorems 1.1 and 1.2 , the ratio of tiling generating functions of $Q_{x}\left(\left(a_{i}\right)_{i=1}^{m}\right)$ and its sibling $Q_{y}\left(\left(a_{i}\right)_{i=1}^{m}\right)$ is always given by a simple product formula (even though each tilling generating function is not a simple product). 

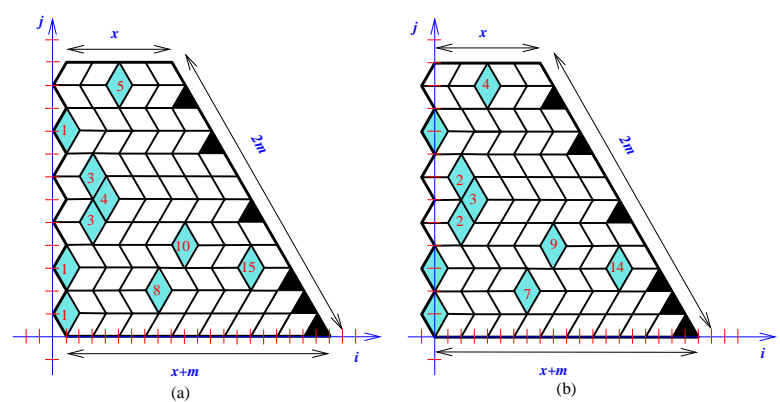

Figure 5: Assigning weights to the lozenges of a quartered hexagon. The shaded lozenges passed by the $j$-axis are weighted by $\frac{1}{2}$; the ones with label $n$ are weighted by $\frac{q^{n}+q^{-n}}{2}$.

Theorem 1.3. For non-negative integers $x, m$ and an increasing sequence $\boldsymbol{a}=\left(a_{i}\right)_{i=1}^{m}$ of positive integers between 1 and $2 m$, the region $Q_{x}\left(\left(a_{i}\right)_{i=1}^{m}\right)$ is tile-able if and only if the region $Q_{y}\left(\left(a_{i}\right)_{i=1}^{m}\right)$ is tile-able. Moreover, we have

$$
\frac{\mathrm{T}\left(Q_{x}\left(\left(a_{i}\right)_{i=1}^{m}\right)\right)}{\mathrm{T}\left(Q_{y}\left(\left(a_{i}\right)_{i=1}^{m}\right)\right)}=q^{2(y-x)\left(\sum_{i=1}^{m} a_{i}-m^{2}\right)} \prod_{i=1}^{m} \frac{\left(q^{2\left(2 y+a_{i}+1\right)} ; q^{2}\right)_{2 i-a_{i}-1}}{\left(q^{2\left(2 x+a_{i}+1\right)} ; q^{2}\right)_{2 i-a_{i}-1}}
$$

whenever $Q_{x}\left(\left(a_{i}\right)_{i=1}^{m}\right)$ is tile-able.

Next, we consider a variation of the quartered region above. The new weighted region has the same shape as the one in Theorem 1.3. The only difference is in the lozenge-weights. We now re-assign the weights to the lozenges, as in Figure 5(b). One should note that the new $j$-axis is one unit to the right of the $j$-axis in Figure $5(\mathrm{a})$. As a consequence, our region now has some vertical lozenges intersected by the $j$-axis. The vertical lozenge with center at the point $(i, j)$ in the new coordinate system is still weighted by $\frac{q^{i}+q^{-i}}{2}$, with one exception: the vertical lozenges intersected by the $j$-axis are weighted by $1 / 2\left(\right.$ not by $\left.1=\frac{q^{0}+q^{-0}}{2}\right)$. Denote by $Q_{x}^{\prime}\left(\left(a_{i}\right)_{i=1}^{m}\right)$ the new weighted region.

Theorem 1.4. Assume that $x, m$ are non-negative integers and that $\left(a_{i}\right)_{i=1}^{m}$ is an increasing sequence of positive integers between 1 and $2 m$. The region $Q_{x}^{\prime}\left(\left(a_{i}\right)_{i=1}^{m}\right)$ is tile-able if and only if the region $Q_{y}^{\prime}\left(\left(a_{i}\right)_{i=1}^{m}\right)$ is tile-able. Moreover, we have

$$
\frac{\mathrm{T}\left(Q_{x}^{\prime}\left(\left(a_{i}\right)_{i=1}^{m}\right)\right)}{\mathrm{T}\left(Q_{y}^{\prime}\left(\left(a_{i}\right)_{i=1}^{m}\right)\right)}=q^{2(y-x)\left(\sum_{i=1}^{m} a_{i}-m^{2}\right)} \prod_{i=1}^{m} \frac{\left(q^{2\left(2 y+a_{i}\right)} ; q^{2}\right)_{2 i-a_{i}-1}}{\left(q^{2\left(2 x+a_{i}\right)} ; q^{2}\right)_{2 i-a_{i}-1}}
$$

if $Q_{x}^{\prime}\left(\left(a_{i}\right)_{i=1}^{m}\right)$ is tile-able.

Remark 1.1 (Combinatorial reciprocity phenomenon). The ratio in Theorem 1.4 is obtained from the one in Theorem 1.3 by replacing $x$ by $x-1 / 2$ and $y$ by $y-1 / 2$. This reminds us of the "combinatorial reciprocity phenomenon": even though the regions $Q_{x}\left(\left(a_{i}\right)_{i=1}^{m}\right)$ and $Q_{y}\left(\left(a_{i}\right)_{i=1}^{m}\right)$ are not defined when $x$ and $y$ are halfintegers, the formula of the ratio of their tiling generating functions gives the "numbers" of combinatorial objects of a different sort when evaluated at half-integers. It would be interesting to find a direct explanation for this, i.e., an explanation without requiring the calculation of the tiling generating functions. For more discussions about the combinatorial reciprocity phenomenon, we refer the reader to, e.g. [1, 23, 24].

Remark 1.2. It is worth noticing that Fulmek recently re-proves Theorems 1.1 and 1.3 using lattice path combinatorics and a special matrix factorization in [12].

\section{Preliminaries}

\section{$2.1 \quad$ Tile-ability}

As mentioned in the previous section, the semi-hexagons with dents on both sides may not have any tilings in general. It is not hard to obtain the following tile-ability for these semi-hexagons by using the correspondence between lozenge tilings and non-intersecting lattice paths.

Lemma 2.1. Assume that $x, m, n$ are non-negative integers, and $\left(a_{i}\right)_{i=1}^{m}$ and $\left(b_{j}\right)_{j=1}^{n}$ are two increasing sequences of positive integers between 1 and $m+n$. Then $S_{x}\left(\left(a_{i}\right)_{i=1}^{m} ;\left(b_{j}\right)_{j=1}^{n}\right)$ is tile-able if and only if

$$
\left|\left\{a_{i}\right\}_{i=1}^{m} \cap[t]\right|+\left|\left\{b_{j}\right\}_{j=1}^{n} \cap[t]\right| \leq t,
$$


for any $t=1,2, \ldots, m+n$, where we use the notation $[t]$ for the set of the first $t$ positive integers $\{1,2, \ldots, t\}$, and where $|A|$ denotes the cardinality of a finite set $A$.

Similar to the case of the semi-hexagons, the quartered hexagon $Q_{x}\left(\left(a_{i}\right)_{i=1}^{m}\right)$ may have no tiling. The following lemma provides a condition for the tile-ability of this region.

Lemma 2.2. Assume that $x, m$ are non-negative integers and that $\left(a_{i}\right)_{i=1}^{m}$ is an increasing sequence of positive integers between 1 and $2 m$. Then $Q_{x}\left(\left(a_{i}\right)_{i=1}^{m}\right)$ is tile-able if and only if

$$
\left|\left\{a_{i}\right\}_{i=1}^{m} \cap[2 t]\right| \leq t
$$

for any $t=1,2, \ldots, m$.

\subsection{Kuo Condensation and Region-splitting Lemma}

In the early 2000s, Eric H. Kuo [14] proved several combinatorial interpretations of the well-known Dodgson condensation in linear algebra [9]. Kuo condensation has become a powerful tool in the field of enumeration of tilings.

A perfect matching of a simple graph is a collection of disjoint edges that cover all vertices of the graph. We use the notation $\mathrm{M}(G)$ for the weighted sum of the perfect matchings of the weighted graph $G$, where the weight of a perfect matching is the product of the weights of its edges. We call $\mathrm{M}(G)$ the matching generating function of $G$.

There is a one-to-one correspondence between tilings of a region $R$ in the triangular lattice and perfect matchings of its (planar) dual graph $G$ (i.e., the graph whose vertices are the unit triangles in $R$ and whose edges connect precisely two unit triangles sharing an edge). Each edge of the dual graph inherits the weight of the corresponding lozenge in the region. In particular, we have $\mathrm{T}(R)=\mathrm{M}(G)$.

We will employ the following three versions of the Kuo condensation in our proofs.

Lemma 2.3 (Theorem 5.1 in [14]). Let $G=\left(V_{1}, V_{2}, E\right)$ be a weighted plane bipartite graph in which $\left|V_{1}\right|=\left|V_{2}\right|$. Let vertices $u, v, w, s$ appear on a face of $G$, in that order. If $u, w \in V_{1}$ and $v, s \in V_{2}$, then

$$
\mathrm{M}(G) \mathrm{M}(G-\{u, v, w, s\})=\mathrm{M}(G-\{u, v\}) \mathrm{M}(G-\{w, s\})+\mathrm{M}(G-\{u, s\}) \mathrm{M}(G-\{v, w\}) .
$$

Lemma 2.4 (Theorem 5.3 in [14]). Let $G=\left(V_{1}, V_{2}, E\right)$ be a weighted plane bipartite graph in which $\left|V_{1}\right|=$ $\left|V_{2}\right|+1$. Let vertices $u, v, w, s$ appear on a face of $G$, in that order. If $u, v, w \in V_{1}$ and $s \in V_{2}$, then

$$
\mathrm{M}(G-\{v\}) \mathrm{M}(G-\{u, w, s\})=\mathrm{M}(G-\{u\}) \mathrm{M}(G-\{v, w, s\})+\mathrm{M}(G-\{w\}) \mathrm{M}(G-\{u, v, s\}) .
$$

Lemma 2.5 (Theorem 5.4 in [14]). Let $G=\left(V_{1}, V_{2}, E\right)$ be a weighted plane bipartite graph in which $\left|V_{1}\right|=$ $\left|V_{2}\right|+2$. Let vertices $u, v, w, s$ appear on a face of $G$, in that order. If $u, v, w, s \in V_{1}$, then

$$
\mathrm{M}(G-\{u, w\}) \mathrm{M}(G-\{v, s\})=\mathrm{M}(G-\{u, v\}) \mathrm{M}(G-\{w, s\})+\mathrm{M}(G-\{u, s\}) \mathrm{M}(G-\{v, w\}) .
$$

A forced lozenge of the region $R$ is a lozenge contained in any tilings of $R$. Assume that we remove $k$ forced lozenges $l_{1}, l_{2}, \ldots, l_{k}$ from $R$ and get a new region $R^{\prime}$, then we have

$$
\mathrm{T}(R)=\left(\prod_{i=1}^{k} \mathrm{wt}\left(l_{i}\right)\right) \cdot \mathrm{T}\left(R^{\prime}\right),
$$

where $\operatorname{wt}\left(l_{i}\right)$ is the weight of the removed lozenge $l_{i}$, for $i=1,2, \ldots, k$.

A region in the triangular lattice must have the same number of up-pointing and down-pointing unit triangles to admit a tiling. We call such a region balanced. The following simple lemma is especially useful when enumerating tilings.

Lemma 2.6 (Region-splitting Lemma [15,16]). Assume $R$ is a balanced region and $Q$ is a subregion of $R$ satisfying the following two conditions:

1. The unit triangles in $Q$ lying on the boundary between $Q$ and $R \backslash Q$ have the same orientation (all are up-pointing or all are down-pointing);

2. $Q$ is balanced.

Then we have $\mathrm{T}(R)=\mathrm{T}(Q) \cdot \mathrm{T}(R \backslash Q)$. 


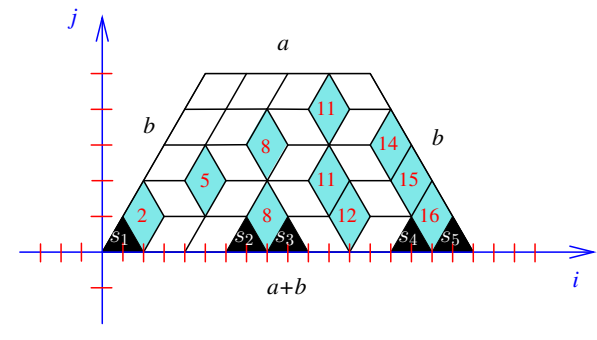

(a)

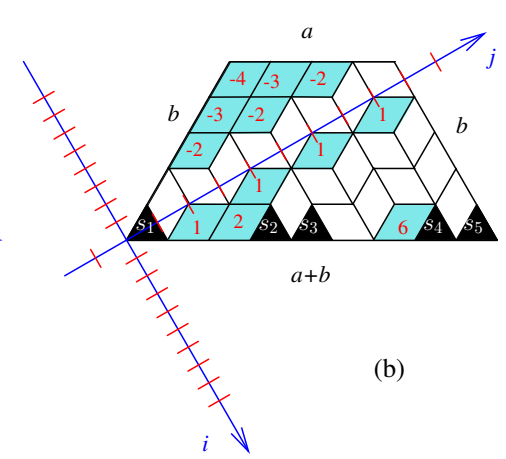

Figure 6: Two ways to assign weights to the lozenges of a semi-hexagon with dents on the base. The shaded lozenges passed by the $j$-axis are weighted by $\frac{X+Y}{2}$, the ones with label $k$ are weighted by $\frac{X q^{k}+Y q^{-k}}{2}$.

\subsection{Four basic enumerations}

We also need the following four basic enumerations for our proofs.

We consider a semi-hexagon of side-lengths $a, b, a+b, b$. We remove $b$ up-pointing unit triangles from the base at the positions $s_{1}, s_{2}, \ldots, s_{b}$ as they appear from left to right. We now assign weights to the vertical lozenges of the dented semi-hexagon, as in Figure 6(a). In particular, the vertical lozenges with center at the point $(i, j)$ are weighted by $\frac{X q^{i}+Y q^{-i}}{2}$. All other lozenges are weighted by 1 . Denote by $S_{a, b}\left(s_{1}, s_{2}, \ldots, s_{b}\right)$ the resulting weighted region.

It is worth noticing that the tiling number of this dented semi-hexagon was first provided by Cohn, Larsen, and Propp [7, Proposition 2.1]. A weighted version of Cohn-Larsen-Propp's result can be found in [25, pp. 374375], in terms of the column-strict plane partitions (or reverse semi-standard Young tableaux). The following lemma was proved implicitly in [2].

Lemma 2.7. For non-negative integers $a, b$, and an increasing sequence $\left(s_{i}\right)_{i=1}^{b}$ of positive integers between 1 and $a+b$, we have

$$
\mathrm{T}\left(S_{a, b}\left(s_{1}, s_{2}, \ldots, s_{b}\right)\right)=2^{-\left(\begin{array}{c}
b \\
2
\end{array}\right)} q^{\sum_{i=1}^{b}(b-1)\left(i+1-2 s_{i}\right)} \prod_{1 \leq i<j \leq b} \frac{q^{2 s_{j}}-q^{2 s_{i}}}{q^{2 j}-q^{2 i}} \prod_{i=1}^{b} \prod_{j=1}^{i-1}\left(q^{2\left(s_{i}+s_{j}-2\right)} X+Y\right) .
$$

Next, we consider a variation of the weighted region above. We now weigh the lozenges of the semi-hexagon differently, as in Figure 6(b). Denote by $S_{a, b}^{\prime}\left(s_{1}, s_{2}, \ldots, s_{b}\right)$ the new weighted region. We have the following counterpart of Lemma 2.7.

Lemma 2.8. For non-negative integers $a, b$, and an increasing sequence $\left(s_{i}\right)_{i=1}^{b}$ of positive integers between 1 and $a+b$

$$
\mathrm{T}\left(S_{a, b}^{\prime}\left(s_{1}, s_{2}, \ldots, s_{b}\right)\right)=2^{\sum_{i=1}^{b}\left(i-s_{i}\right)} q^{\sum_{i=1}^{b} \frac{\left(i-s_{i}\right)\left(s_{i}-3+i\right)}{2}} \prod_{1 \leq i<j \leq b} \frac{q^{2 s_{j}}-q^{2 s_{i}}}{q^{2 j}-q^{2 i}} \prod_{i=1}^{b} \prod_{j=1}^{s_{i}-i}\left(q^{2(i+j-b-1)} X+Y\right) .
$$
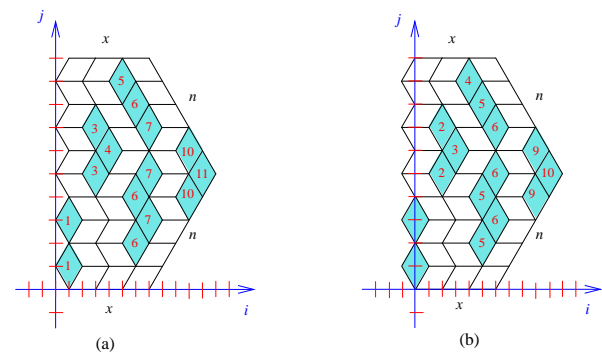

Figure 7: Two ways to assign weights to lozenges of a halved hexagon. The shaded lozenges passed by the $j$-axis are weighted by $\frac{1}{2}$, the ones with label $k$ are weighted by $\frac{q^{k}+q^{-k}}{2}$.

"We only consider regions in the triangular lattice in this paper. From now on, we will use the term "region(s)" to mean "region(s) in the triangular lattice." 
Next, we consider a pentagonal region with side-lengths $x, n, n, x, 2 n$, whose vertical right side runs along a zigzag path with $2 n$ steps. We now assign the weight to lozenges of the region as in Figure 7(a): the vertical lozenges with center at the point $(i, j)$ are weighted by $\frac{q^{i}+q^{-i}}{2}$; other lozenges are all weighted by 1 . Denote by $P_{x, n}$ the resulting weighted region. We usually call $P_{x, n}$ a (weighted) halved hexagon, as it can be viewed as half of a symmetric hexagon of side-lengths $2 x+1, n, n, 2 x+1, n, n$ divided by a vertical zigzag cut.

The $q$-integer $[n]_{q}$ is defined as $[n]_{q}=1+q+\cdots+q^{n-1}$, where $[0]_{q}=0$. Then the $q$-factorial is defined to be the product of $q$-integers: $[n]_{q} !=[1]_{q}[2]_{q} \cdots[n]_{q}$, where $[0]_{q} !=1$.

Lemma 2.9. Assume that $x, n$ are non-negative integers. Then we have

$$
\mathrm{T}\left(P_{x, n}\right)=\frac{2^{-n^{2}} q^{-\sum_{i=1}^{n}(2 i-1)(2 x+i)}}{[1]_{q^{2}} ![3]_{q^{2}} ! \cdots[2 n-1]_{q^{2}} !} \prod_{i=1}^{n}[4(x+i)]_{q^{2}} \prod_{1 \leq i<j \leq n}[2(2 x+i+j)]_{q^{2}}[2(j-i)]_{q^{2}} .
$$

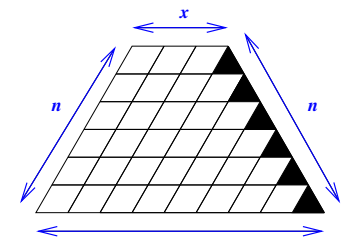

$x+n$
$($ a)

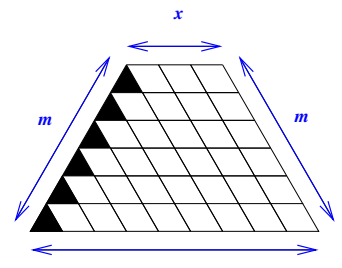

(b)

Figure 8: Two base cases in the proof of Theorem 1.1: (a) the case $m=0$ and (b) the case $n=0$.

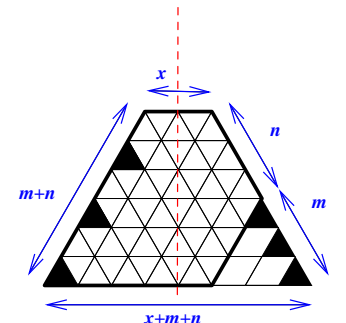

(a)

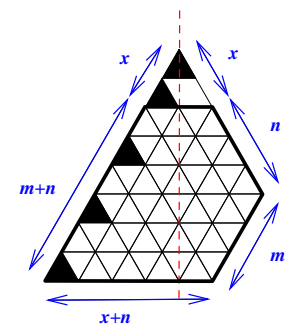

(b)

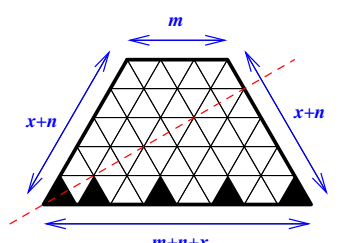

(c)

Figure 9: The case $t=0$ in the proof of Theorem 1.1.

We also consider a variant of $P_{x, n}$ obtained by re-assigning the lozenge-weights as in Figure $5(\mathrm{~b})$. In particular, the vertical lozenges with center at the point $(i, j)$ are still weighted by $\frac{q^{i}+q^{-i}}{2}$, except for the ones intersected by the $j$-axis, which are weighted by $1 / 2\left(\right.$ not $\left.1=\frac{q^{0}+q^{-0}}{2}\right)$. Denote by $P_{x, n}^{\prime}$ the new weighted region.

Lemma 2.10. Assume that $x, n$ are non-negative integers. Then we have

$$
\mathrm{T}\left(P_{x, n}^{\prime}\right)=\frac{2^{-n^{2}} q^{-\sum_{i=1}^{n}(2 i-1)(2 x+i-1)}}{[1]_{q^{2}} ![3]_{q^{2}} ! \cdots[2 n-1]_{q^{2}} !} \prod_{i=1}^{n}[4(x+i)-2]_{q^{2}} \prod_{1 \leq i<j \leq n}[2(2 x+i+j-1)]_{q^{2}}[2(j-i)]_{q^{2}} .
$$

To the best of the author's knowledge, there are no references for the proofs of Lemmas 2.8, 2.9, and 2.10 in the literature. For the completeness of the paper, we will provide the proofs of these lemmas in the Appendix.

\section{Proofs of Main Theorems}

Proof of Theorem 1.1. By Lemma 2.1, the region $S_{x}\left(\left(a_{i}\right)_{i=1}^{m} ;\left(b_{j}\right)_{j=1}^{n}\right)$ is tile-able if and only if the region $S_{y}\left(\left(a_{i}\right)_{i=1}^{m} ;\left(b_{j}\right)_{j=1}^{n}\right)$ is tile-able. 


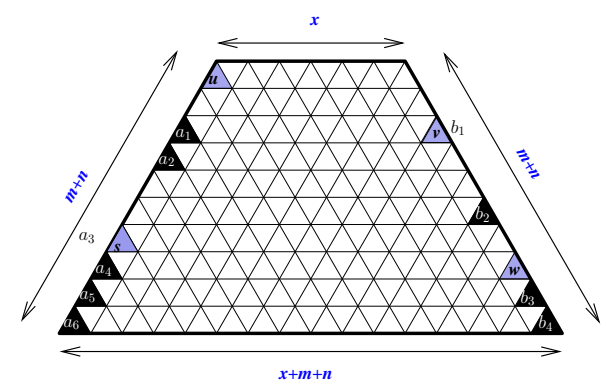

Figure 10: How to apply Kuo condensation to a semi-hexagon with dents on two sides.

Next, we assume that $S_{x}\left(\left(a_{i}\right)_{i=1}^{m} ;\left(b_{j}\right)_{j=1}^{n}\right)$ has a tiling. We rewrite identity (2) as

$$
\mathrm{T}\left(S_{x}\left(\left(a_{i}\right)_{i=1}^{m} ;\left(b_{j}\right)_{j=1}^{n}\right)\right)=f_{x, y}\left(\left(a_{i}\right)_{i=1}^{m} ;\left(b_{j}\right)_{j=1}^{n}\right) \cdot \mathrm{T}\left(S_{y}\left(\left(a_{i}\right)_{i=1}^{m} ;\left(b_{j}\right)_{j=1}^{n}\right)\right),
$$

where $f_{x, y}\left(\left(a_{i}\right)_{i=1}^{m} ;\left(b_{j}\right)_{j=1}^{n}\right)$ denotes the expression on the right-hand side of $(2)$.

We prove (17) by induction on the statistic $p:=m+2 n+t$, where $t=\sum_{i=1}^{n}\left((m+i)-b_{i}\right)$. The base cases are the situations when at least one of the perimeters $m, n, t$ is equal to 0 .

If $m=0$, then our two semi-hexagons $S_{x}(\mathbf{a}, \mathbf{b})$ and $S_{y}(\mathbf{a}, \mathbf{b})$ have exactly 1 tiling as shown in Figure 8(a). In this case, identity (17) becomes " $1=1$." The case $n=0$ is similar (illustrated in Figure $8(\mathrm{~b})$ ).

If $t=0$, then all $b$-dents of $S_{x}\left(\left(a_{i}\right)_{i=1}^{m} ;\left(b_{j}\right)_{j=1}^{n}\right)$ are clustering to the lower-right corner. By removing forced lozenges, we get a pentagonal region with dents on the left side (illustrated by the region restricted by the bold contour in Figure 9(a)). The resulting region has the same tiling generating function as the region in Figure 9(b). (The two regions differ by only several forced lozenges with weight 1.) We now $120^{\circ}$-rotate this region to get a weighted semi-hexagon in Lemma 2.7 (see Figure 9(c)). This way, we obtain an explicit formula for the tiling generating function of the semi-hexagon $S_{x}\left(\left(a_{i}\right)_{i=1}^{m} ;\left(b_{j}\right)_{j=1}^{n}\right)$, and similarly for $S_{y}\left(\left(a_{i}\right)_{i=1}^{m} ;\left(b_{j}\right)_{j=1}^{n}\right)$. Identity (17) now follows directly from Lemma 2.7 in this case.
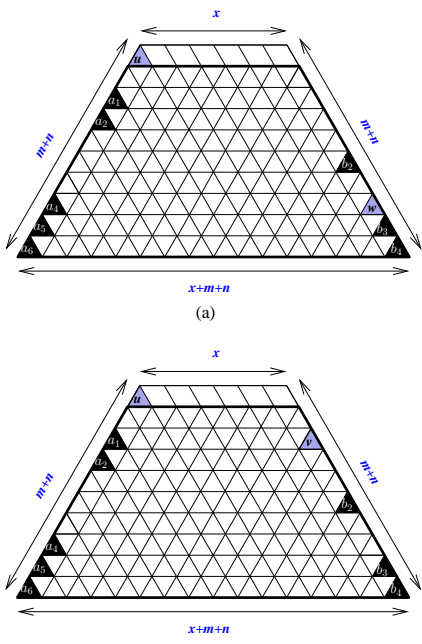

(c)

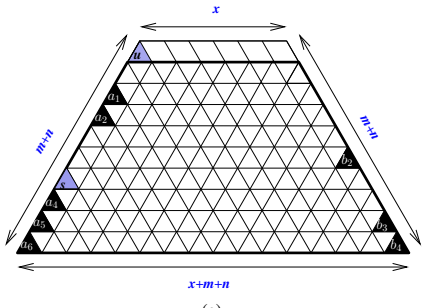

(e)

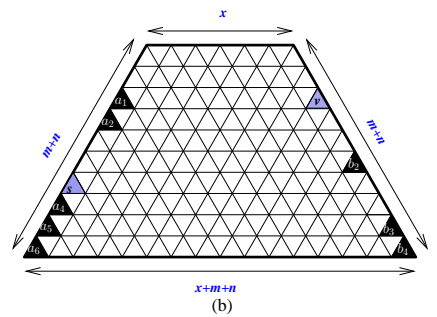

(b)

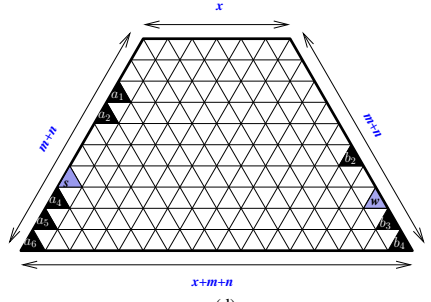

(d)

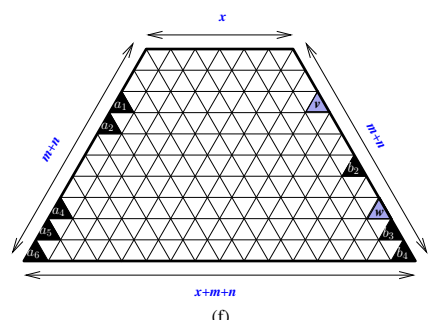

Figure 11: Obtaining a recurrence for tiling generating functions of the semi-hexagons with dents on two sides.

For the induction step, we assume that $m, n, t$ are all positive and that (17) holds for any pair of semihexagons whose $p$-statistic is strictly less than $m+2 n+t$.

By the tile-ability of the semi-hexagons in Lemma 2.1, at least one of $a_{1}$ and $b_{1}$ is strictly greater than 1. Without loss of generality, we assume that $a_{1}>1$. If $b_{1}=1$, then one can remove forced lozenges on the top 
of the two semi-hexagons to get two "smaller" $\|$ semi-hexagons of the same type. Then (17) follows from the induction hypothesis. Therefore, we can also assume that $b_{1}>1$.

When $a_{1}, b_{1}>1$, we will show that the expressions on both sides of (17) satisfy the same recurrence. Then the theorem follows from the induction principle.

Assume that $l$ is the largest index such that there is no $a$-dent at the position $a_{l}-1$ on the left side of $S$. We consider the region $R$ obtained by filling the dents at the positions $a_{l}$ and $b_{1}$ in $S$ (see Figure 10 for an example when $l=3$ ). We apply Kuo condensation in Lemma 2.5 to the dual graph $G$ of $R$ with the four vertices $u, v, w, s$ corresponding to the shaded unit triangles of the same label. More precisely, the $u$-triangle is the up-pointing triangle on the upper-left corner of $S$, the $v$-triangle is at the position $b_{1}$, the $w$-triangle is at the last non-dent position on the right side of $S$, and the $s$-triangle is at the position $a_{l}$. Let $\alpha$ denote the position of the $w$-triangle.

We consider the region corresponding to graph $G-\{u, w\}$ (as shown in Figure 11(a)). The removal of the $u$ and $w$-triangles yields forced lozenges (with weight 1 ) on the top of the region. Removal of these forced lozenges gives a new semi-hexagon, namely $S_{x+1}\left(\left(\mathbf{a}-a_{l}\right)^{*} ;\left(\mathbf{b}-b_{1}+\alpha\right)^{*}\right)$. Here, we use the notations $\mathbf{a}-c$ and $\mathbf{a}+d$ for the sequences obtained from $\mathbf{a}=\left(a_{i}\right)_{i=1}^{m}$ by excluding the term $c$ and by including the term $d$ (and rearranging in increasing order), respectively. We also use the notation $\mathbf{a}^{*}$ for the sequence obtained from sequence $\mathbf{a}$ by subtracting 1 from each of its terms. By the correspondence between tilings and perfect matchings, we get

$$
\mathrm{M}(G-\{u, w\})=\mathrm{T}\left(S_{x+1}\left(\left(\mathbf{a}-a_{l}\right)^{*} ;\left(\mathbf{b}-b_{1}+\alpha\right)^{*}\right)\right) .
$$

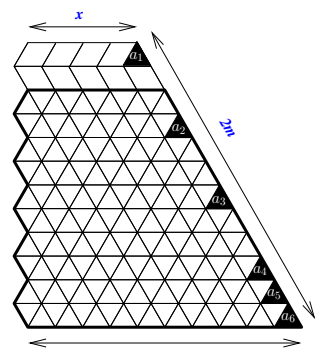

$x+m$
$(a)$

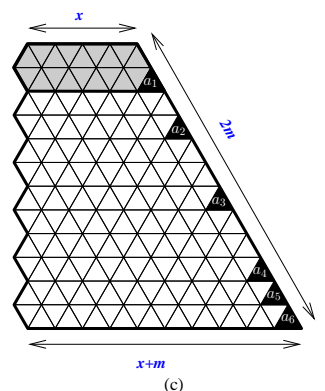

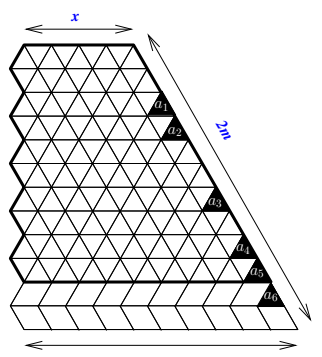

$x+m$
(b)

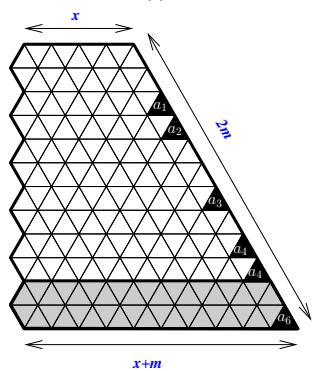

(d)

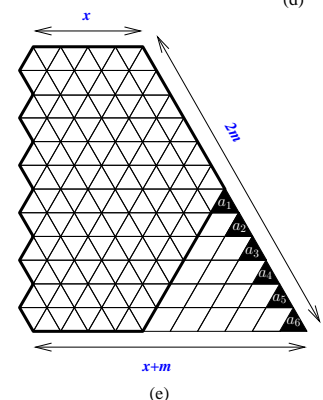

Figure 12: Several special cases in the proof of Theorem 1.3: (a) the case $a_{1}=1$, (b) the case $t=0$, (c) the case $a_{1}=2$, (d) the case $t=1$ (i.e., $a_{m}=2 m$ and $a_{m-1} \leq 2 m-2$ ), and (e) the case $t=m$.

Working similarly based on Figures 11(b)-(f), we get

$$
\begin{gathered}
\mathrm{M}(G-\{v, s\})=\mathrm{T}\left(S_{x}(\mathbf{a} ; \mathbf{b})\right), \quad \mathrm{M}(G-\{u, v\})=\mathrm{T}\left(S_{x+1}\left(\left(\mathbf{a}-a_{l}\right)^{*} ; \mathbf{b}^{*}\right)\right), \\
\mathrm{M}(G-\{u, s\})=\mathrm{T}\left(S_{x+1}\left(\mathbf{a}^{*} ;\left(\mathbf{b}-b_{1}\right)^{*}\right)\right), \quad \mathrm{M}(G-\{v, s\})=\mathrm{T}\left(S_{x}\left(\mathbf{a}-a_{l} ; \mathbf{b}+\alpha\right)\right) .
\end{gathered}
$$

\footnotetext{
"In the rest of this proof, we say that the semi-hexagon $A$ is smaller than the semi-hexagon $B$ if the $p$-statistic of $A$ is less than that of $B$.
} 
Plugging these six equations into the equation in Lemma 2.5, we get the recurrence:

$$
\begin{aligned}
\mathrm{T}\left(S_{x+1}\left(\left(\mathbf{a}-a_{l}\right)^{*} ;\left(\mathbf{b}-b_{1}+\alpha\right)^{*}\right) \mathrm{T}\left(S_{x}(\mathbf{a} ; \mathbf{b})\right)\right. & =\mathrm{T}\left(S_{x+1}\left(\left(\mathbf{a}-a_{l}\right)^{*} ; \mathbf{b}^{*}\right)\right) \mathrm{T}\left(S_{x}\left(\mathbf{a} ; \mathbf{b}-b_{1}+\alpha\right)\right) \\
& +\mathrm{T}\left(S_{x+1}\left(\mathbf{a}^{*} ;\left(\mathbf{b}-b_{1}\right)^{*}\right)\right) \mathrm{T}\left(S_{x}\left(\mathbf{a}-a_{l} ; \mathbf{b}+\alpha\right)\right) .
\end{aligned}
$$

One could verify that the $p$-statistics of these five semi-hexagons are all strictly less than $m+2 n+t$, which is the $p$-statistic of $S$.

To complete the proof, we want to show that the expression $f_{x, y}(\mathbf{a} ; \mathbf{b}) \cdot \mathrm{T}\left(S_{y}(\mathbf{a} ; \mathbf{b})\right)$ on the right-hand side of (17) also satisfies the same recurrence. Equivalently, we need to verify that

$$
\begin{aligned}
A \cdot \mathrm{T}\left(S_{y+1}\left(\left(\mathbf{a}-a_{l}\right)^{*} ;\left(\mathbf{b}-b_{1}+\alpha\right)^{*}\right) \mathrm{T}\left(S_{y}(\mathbf{a} ; \mathbf{b})\right)\right. & =B \cdot \mathrm{T}\left(S_{y+1}\left(\left(\mathbf{a}-a_{l}\right)^{*} ; \mathbf{b}^{*}\right)\right) \mathrm{T}\left(S_{y}\left(\mathbf{a} ; \mathbf{b}-b_{1}+\alpha\right)\right) \\
& +C \cdot \mathrm{T}\left(S_{y+1}\left(\mathbf{a}^{*} ;\left(\mathbf{b}-b_{1}\right)^{*}\right)\right) \mathrm{T}\left(S_{y}\left(\mathbf{a}-a_{l} ; \mathbf{b}+\alpha\right)\right),
\end{aligned}
$$

where $A=f_{x+1, y+1}\left(\left(\mathbf{a}-a_{l}\right)^{*} ;\left(\mathbf{b}-b_{1}+\alpha\right)^{*}\right) \cdot f_{x, y}(\mathbf{a} ; \mathbf{b}), B=f_{x+1, y+1}\left(\left(\mathbf{a}-a_{l}\right)^{*} ; \mathbf{b}^{*}\right) \cdot f_{x, y}\left(\mathbf{a} ; \mathbf{b}-b_{1}+\alpha\right)$, and $C=f_{x+1, y+1}\left(\mathbf{a}^{*} ;\left(\mathbf{b}-b_{1}\right)^{*}\right) \cdot f_{x, y}\left(\mathbf{a}-a_{l} ; \mathbf{b}+\alpha\right)$.

It is routine to verify that $A=B=C$. Canceling out $A, B, C$ terms, we now only need to verify that

$$
\begin{aligned}
\mathrm{T}\left(S_{y+1}\left(\left(\mathbf{a}-a_{l}\right)^{*} ;\left(\mathbf{b}-b_{1}+\alpha\right)^{*}\right) \mathrm{T}\left(S_{y}(\mathbf{a} ; \mathbf{b})\right)\right. & =\mathrm{T}\left(S_{y+1}\left(\left(\mathbf{a}-a_{l}\right)^{*} ; \mathbf{b}^{*}\right)\right) \mathrm{T}\left(S_{y}\left(\mathbf{a} ; \mathbf{b}-b_{1}+\alpha\right)\right) \\
& +\mathrm{T}\left(S_{y+1}\left(\mathbf{a}^{*} ;\left(\mathbf{b}-b_{1}\right)^{*}\right)\right) \mathrm{T}\left(S_{y}\left(\mathbf{a}-a_{l} ; \mathbf{b}+\alpha\right)\right) .
\end{aligned}
$$

However, this recurrence follows directly from recurrence (18) by simply replacing $x$ with $y$. This finishes our proof.

One could prove Theorem 1.2 in the same way as Theorem 1.1, using Lemma 2.8. We leave this proof as an exercise to the reader. We now prove Theorem 1.3.

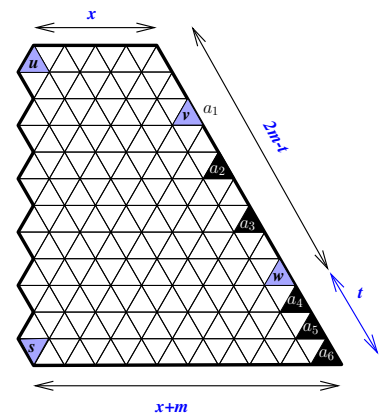

Figure 13: How to apply Kuo condensation to the quartered hexagon.

Proof of Theorem 1.3. By Lemma 2.2, the region $Q_{x}=Q_{x}(\mathbf{a})$ has a tiling if and only if the region $Q_{y}=Q_{y}(\mathbf{a})$ has a tiling, where $\mathbf{a}=\left(a_{i}\right)_{i=1}^{m}$.

Next, we assume that $Q_{x}$ is tile-able. We reformulate our identity (5) as

$$
\mathrm{T}\left(Q_{x}\left(\left(a_{i}\right)_{i=1}^{m}\right)\right)=g_{x, y}\left(\left(a_{i}\right)_{i=1}^{m}\right) \cdot \mathrm{T}\left(Q_{y}\left(\left(a_{i}\right)_{i=1}^{m}\right)\right),
$$

where $g_{x, y}\left(\left(a_{i}\right)_{i=1}^{m}\right)$ is the express on the right-hand side of $(5)$.

Let $t$ be the size of the maximal cluster of dents attaching to the lower-right corner of the region $Q_{x}$. We prove (21) by induction on the statistic $h:=2 m-t$, the base cases are the situations when $m=0,1$ and $t=m$.

If $m=0$, then our identity simply becomes " $1=1$." If $m=1$, then there are only two cases $\mathbf{a}=\left(a_{1}\right)=(1)$ or (2). If $a_{1}=1$, then both $Q_{x}\left(\left(a_{1}\right)\right)$ and $Q_{y}\left(\left(a_{1}\right)\right)$ has tiling generating function 1 , and our identity is obviously true. If $a_{1}=2$, then our region is exactly the halved hexagon $P_{x, 1}$, and (21) follows from Lemma 2.9 .

If $t=m$, then all of the dents are clustering to the lower-right corner of the region. Then our region, after removed forced lozenges, becomes a halved hexagon $P_{x, m}$ in Lemma 2.9 (see Figure 12(e)). Again, (21) follows from Lemma 2.9 .

For the induction step, we assume that $m \geq 2$ and $t<m$, and that identity (21) holds for any pair of quartered hexagons whose $h$-statistics are strictly less than $2 m-t$.

First, we will show below that one could assume that $a_{1} \geq 3$ and $t \geq 2$. Indeed, if $a_{1}=1$, then $a_{2} \geq 3$ by the tile-ability in Lemma 2.2. Then we get forced lozenges along the first and second rows of unit triangles in $Q_{x}$ (see Figure 12(a)). After removing these forced lozenges (whose weights are all 1), we get a 'smaller'**

\footnotetext{
** Similar to the case of dented semi-hexagons, we say a quartered hexagon is "smaller" than another quartered hexagon if its $h$-statistic is less than that of the latter one.
} 


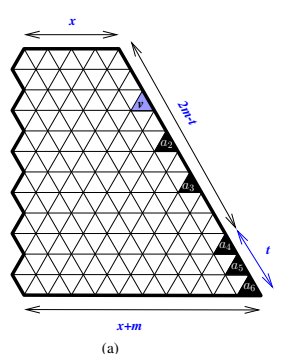

(a)

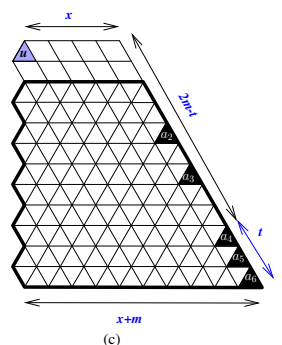

(c)

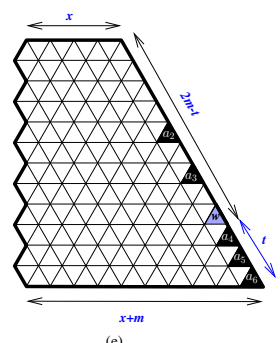

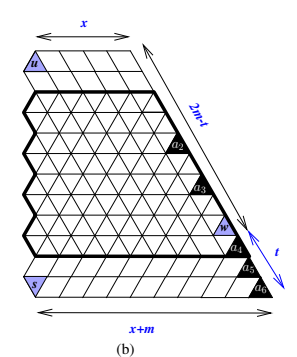
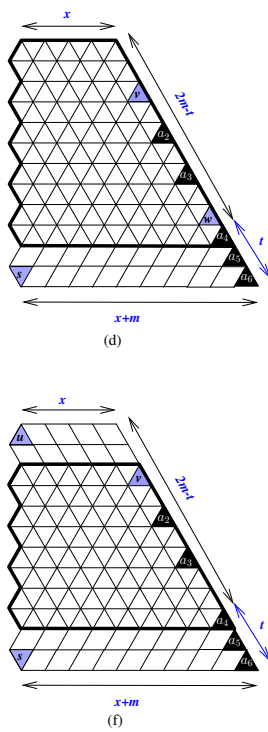

Figure 14: Obtaining a recurrence for the tiling generating functions of the quartered hexagons.

quartered hexagon with the same tiling generating function. We can do similarly for $Q_{y}$, and (21) follows from the induction hypothesis. We do similarly for the case $t=0$ (illustrated in Figure 12(b)).

If $a_{1}=2$, then we can apply the Region-splitting Lemma 2.6 to split the region $Q_{x}$ into two smaller quartered hexagons as in Figure 12(c). (The cut is at level 2 from the top of $Q_{x}$; the top portion is shaded.) Do similarly for $Q_{y}$, and (21) follows from the Region-splitting Lemma 2.6 and the induction hypothesis. We do similarly for the case $t=1$ (see Figure 12(d)).

When $a_{1} \geq 3$ and $t \geq 2$, we will use Kuo condensation in Lemma 2.4 to show that the expressions on both sides of (21) satisfy the same recurrence.

We apply Kuo condensation in Lemma 2.4 to the dual graph $G$ of $R$, the region obtained from $Q_{x}$ by filling the top dent, with the four vertices $u, v, w, s$ corresponding to the shaded unit triangles in Figure 13. In particular, the $v$-triangle is the up-pointing triangle at the position $a_{1}$, and the $w$-triangle is at the last non-dent position $\beta$ on the right side of $R$. Similar to the proof of Theorem 1.1, Figure 14 tells us that

$$
\begin{aligned}
\mathrm{T}\left(Q_{x}(\mathbf{a})\right) \mathrm{T}\left(Q_{x+1}\left(\left(\left(a_{i}\right)_{i=2}^{m-2}+\beta\right)^{* *}\right)\right. & =\mathrm{T}\left(Q_{x+1}\left(\left(a_{i}\right)_{i=2}^{m}\right)^{* *}\right) \mathrm{T}\left(Q_{x}\left(\left(a_{i}\right)_{i=1}^{m-2}+\beta\right)\right) \\
& +\mathrm{T}\left(Q_{x}\left(\left(a_{i}\right)_{i=2}^{m}+\beta\right)\right) \mathrm{T}\left(Q_{x+1}\left(\left(\left(a_{i}\right)_{i=1}^{m-2}\right)^{* *}\right),\right.
\end{aligned}
$$

where we use the notation $\mathbf{s}^{* *}$ for the sequence obtained by subtracting 2 from each term of $\mathbf{s}$.

To finish the proof, we need to show that the expression $g_{x, y}(\mathbf{a}) \cdot \mathrm{T}\left(Q_{y}\left(\left(a_{i}\right)_{i=1}^{m}\right)\right)$ on the right-hand side of (21) also satisfies recurrence (22) above. Equivalently, we need to verify that

$$
\begin{aligned}
A^{\prime} \cdot \mathrm{T}\left(Q_{y}(\mathbf{a})\right) \mathrm{T}\left(Q_{y+1}\left(\left(\left(a_{i}\right)_{i=2}^{m-2}+\beta\right)^{* *}\right)\right. & =B^{\prime} \cdot \mathrm{T}\left(Q_{y+1}\left(\left(a_{i}\right)_{i=2}^{m}\right)^{* *}\right) \mathrm{T}\left(Q_{y}\left(\left(a_{i}\right)_{i=1}^{m-2}+\beta\right)\right) \\
& +C^{\prime} \cdot \mathrm{T}\left(Q_{y}\left(\left(a_{i}\right)_{i=2}^{m}+\beta\right)\right) \mathrm{T}\left(Q_{y+1}\left(\left(\left(a_{i}\right)_{i=1}^{m-2}\right)^{* *}\right),\right.
\end{aligned}
$$

where $\left.A^{\prime}=g_{x, y}(\mathbf{a}) \cdot g_{x+1, y+1}\left(\left(\left(a_{i}\right)_{i=2}^{m-2}+\beta\right)^{* *}\right), B^{\prime}=g_{x+1, y+1}\left(\left(a_{i}\right)_{i=2}^{m}\right)^{* *}\right) \cdot g_{x, y}\left(\left(\left(a_{i}\right)_{i=1}^{m-2}+\beta\right)\right.$, and $C^{\prime}=$ $g_{x, y}\left(\left(a_{i}\right)_{i=2}^{m}+\beta\right) \cdot g_{x+1, y+1}\left(\left(\left(a_{i}\right)_{i=1}^{m-2}\right)^{* *}\right)$.

By definition, one could routinely verify that $A^{\prime}=B^{\prime}=C^{\prime}$. Then (23) reduces to

$$
\begin{aligned}
\mathrm{T}\left(Q_{y}(\mathbf{a})\right) \mathrm{T}\left(Q_{y+1}\left(\left(\left(a_{i}\right)_{i=2}^{m-2}+\beta\right)^{* *}\right)\right. & =\mathrm{T}\left(Q_{y+1}\left(\left(a_{i}\right)_{i=2}^{m}\right)^{* *}\right) \mathrm{T}\left(Q_{y}\left(\left(a_{i}\right)_{i=1}^{m-2}+\beta\right)\right) \\
& +\mathrm{T}\left(Q_{y}\left(\left(a_{i}\right)_{i=1}^{m}+\beta\right)\right) \mathrm{T}\left(Q_{y+1}\left(\left(\left(a_{i}\right)_{i=1}^{m-2}\right)^{* *}\right) .\right.
\end{aligned}
$$


However, this recurrence follows immediately from recurrence (22) by replacing $x$ with $y$. This finishes our proof.

Theorem 1.4 can be proved in the same manner as Theorem 1.3, using Lemma 2.10. Even though the lozenges are weighted differently in Theorems 1.3 and 1.4, the Kuo condensation works essentially the same as the forced lozenges all have weight 1 . We omit the proof of Theorem 1.4 here.

\section{Appendix: Proofs of Lemmas 2.7-2.10}

We first show briefly here the proof of Lemma 2.7. The proof of Lemma 2.8 is essentially the same.

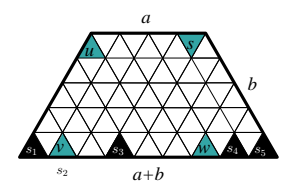

Figure 15: How to apply Kuo condensation to the semi-hexagon with dents on the base.

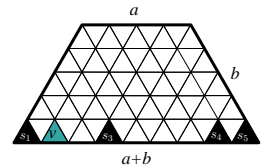

(a)

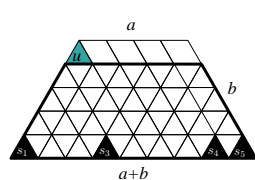

(c)

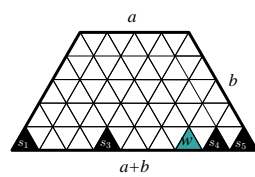

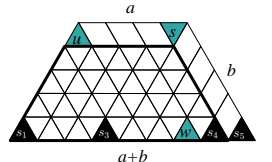

(b)

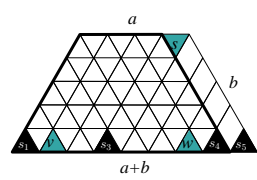

(d)

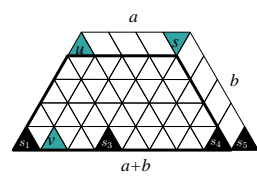

Figure 16: Obtaining recurrence for tiling generating functions of the semi-hexagons with dents on the base.

Proof of Lemma 2.7. We define $t=b-l$, where $l$ is the size of the maximal dent cluster attaching to the lower-right corner of the semi-hexagon $S=S_{a, b}(\mathbf{s})$, where $\mathbf{s}=\left(s_{i}\right)_{i=1}^{b}$. We prove the lemma by induction on $a+b+t$. One could easily verify the base cases when at least one of $a, b, t$ is equal to 0 .

For the induction step, we assume that $a, b, t>0$ and that the lemma holds for any semi-hexagons whose sum of $a-, b-$, and $t$-parameters is strictly less than $a+b+t$. It is easy to see that we can assume $s_{1}=1$ and $s_{b}=a+b$. Otherwise, one can remove forced lozenges from $S$ to obtain a smaller region of the same type, and the lemma follows from the induction hypothesis.

Assume that $s_{k}$ is the first dent position so that there is no dent on the right of its (in particular, $s_{k+1}>$ $\left.s_{k}+1\right)$. We consider the region $R$ obtained from $S$ by filling the $s_{k}$-dent. $R$ has one more up-pointing triangles than down-pointing triangles. We apply Kuo condensation in Lemma 2.4 to the dual graph $G$ of $R$ with the four vertices $u, v, w, s$ as shown in Figure 15. In particular, the $v$-triangle is at the position of $\alpha=s_{k}$, and the $w$-triangle is at the position $\beta=s_{b-t+1}-1$ on the base. Considering the removal of forced lozenges as in Figure 16 , we get the following recurrence

$$
\begin{aligned}
\mathrm{T}\left(S_{a, b}(\mathbf{s})\right) \mathrm{T}\left(S_{a, b-1}\left(\left(s_{i}\right)_{i=1}^{b-1}-\alpha+\beta\right)\right) & =\mathrm{T}\left(S_{a+1, b-1}(\mathbf{s}-\alpha)\right) \mathrm{T}\left(S_{a-1, b}\left(\left(s_{i}\right)_{i=1}^{b-1}+\beta\right)\right) \\
& +\mathrm{T}\left(S_{a, b}(\mathbf{s}-\alpha+\beta)\right) \mathrm{T}\left(S_{a, b-1}\left(\left(s_{i}\right)_{i=1}^{b-1}\right)\right)
\end{aligned}
$$

(The weights of forced lozenges cancel out.) Then the lemma follows from the induction principle.

Next, we show the proof of Lemma 2.9 (Lemma 2.10 can be proved in the same manner). 

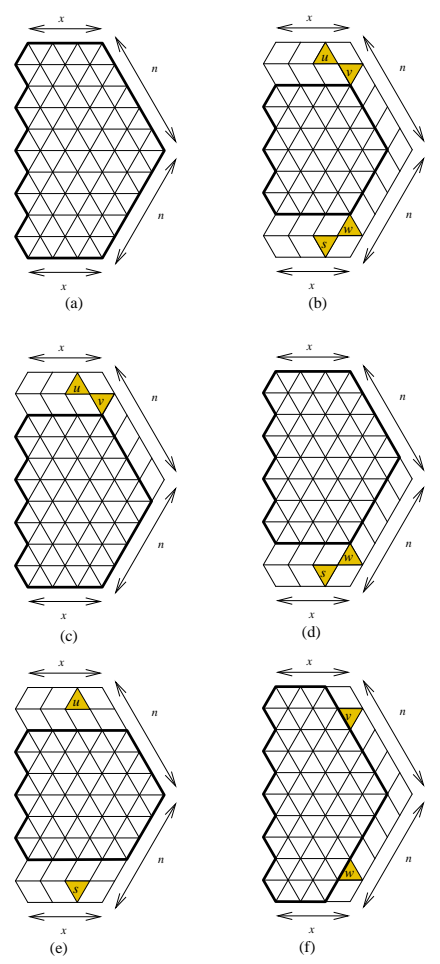

Figure 17: Obtaining recurrence for tiling generating functions of halved hexagons.

Proof of Lemma 2.9. We prove by induction on $x+n$. The base cases are the situations $x=0$ and $n \leq 1$.

When $x=0$, the region $P_{x, n}$ has only one tiling consisting of vertical lozenges; when $n=0$, the region is degenerated. It is easy to verify our identity in these cases. If $n=1$, then our region becomes a hexagon of side-lengths $x, 1,1, x, 1,1$. It is easy to see that the hexagon has exactly $x+1$ tilings; each consists of one vertical lozenge, $x$ left lozenges, and $x$ right lozenges. One could calculate the tilling generating function and easily verify the identity in this case.

When $x>0$ and $n>1$, we apply Kuo condensation in Lemma 2.3 to the dual graph $G$ of the halved hexagon $P_{x, n}$, as shown in Figure 17 . We get the following recurrence:

$$
\mathrm{T}\left(P_{x, n}\right) \mathrm{T}\left(P_{x, n-2}\right)=\left(\frac{q^{2 x+n}+q^{-2 x-n}}{2}\right) \mathrm{T}\left(P_{x, n-1}\right) \mathrm{T}\left(P_{x, n-1}\right)+\mathrm{T}\left(P_{x+1, n-2}\right) \mathrm{T}\left(P_{x-1, n}\right) .
$$

The factor $\frac{q^{2 x+n}+q^{-2 x-n}}{2}$ comes from the weight of the rightmost vertical lozenge in $P_{x, n}$; the weights of all other forced lozenges cancel out. Then the lemma follows from the induction principle.

\section{References}

[1] M. Beck and R. Sanyal, Combinatorial Reciprocity Theorems, AMS Press, Providence, RI, USA, 2018.

[2] A. Borodin, V. Gorin, and E. M. Rains, q-Distributions on boxed plane partitions, Selecta Math. 16 (2010), $731-789$.

[3] S. Byun, A short proof of two shuffing theorems for tilings and a weighted generalization, arXiv:1906.04533.

[4] S. Byun, Lozenge tilings of hexagons with holes on three crossing lines, arXiv:2008.00591.

[5] M. Ciucu and C. Krattenthaler, A dual of MacMahon's theorem on plane partitions, Proc. Natl. Acad. Sci. USA 110 (2013), 4518-4523.

[6] M. Ciucu, T. Lai, and R. Rohatgi, Tilings of hexagons with a removed triad of bowties, J. Combin. Theory Ser. A 178 (2021), 105359.

[7] H. Cohn, M. Larsen, and J. Propp, The shape of a typical boxed plane partition, New York J. Math. 4 (1998), 137-165.

[8] D. Condon, Lozenge tiling function ratios for hexagons with dents on two sides, Electron. J. Combin. 27(3) (2020), \#P3.60.

[9] C.L. Dodgson, Condensation of determinants, Proc. Roy. Soc. London 15 (1866), 150-155. 
[10] N. Elkies, G. Kuperberg, M. Larsen, and J. Propp, Alternating-sign matrices and domino tilings (Part I), J. Algebraic Combin. 1 (1992), 111-132.

[11] N. Elkies, G. Kuperberg, M. Larsen, and J. Propp, Alternating-sign matrices and domino tilings (Part II), J. Algebraic Combin. 1 (1992), 219-234.

[12] M. Fulmek, Generating functions of lozenge tilings for hexagonal regions via nonintersecting lattice paths, Enum. Combin. Appl. 1(3) (2021), \#S2R24.

[13] M. Fulmek, A simple explanation for the "shuffling phenomenon" for lozenge tilings of dented hexagons, Discrete Math. 334(7) (2021), 112396.

[14] E. H. Kuo, Applications of graphical condensation for enumerating matchings and tilings, Theoret. Comput. Sci. 319 (2004), 29-57.

[15] T. Lai, A q-enumeration of a hexagon with four adjacent triangles removed from the boundary, European J. Combin. 64 (2017), 66-87.

[16] T. Lai, A q-enumeration of lozenge tilings of a hexagon with three dents, Adv. Appl. Math. 82 (2017), 23-57.

[17] T. Lai, A shuffling theorem for centrally symmetric lozenge tilings, arXiv:1906.03759.

[18] T. Lai, Tiling enumeration of hexagons with off-central holes, arXiv:1905.07119.

[19] T. Lai, Lozenge tilings of hexagons with central holes and dents, Electron. J. Combin. 27(1) (2020), \#P1.61.

[20] T. Lai, A shuffling theorem for reflectively symmetric lozenge tilings, Discrete Math. 334(7) (2021), 112390.

[21] T. Lai and R. Rohatgi, A shuffling theorem for lozenge tilings of doubly-dented hexagons, arXiv:1905.08311.

[22] P. A. MacMahon, Combinatory Analysis, Volume 2, Cambridge Univ. Press, Cambridge, UK, 1916.

[23] J. Propp, A reciprocity theorem for domino tilings, Electron. J. Combin. 8 (2001), \#R18.

[24] R. Stanley, Combinatorial reciprocity theorems, Adv. Math. 14 (1974), 194-253.

[25] R. Stanley, Enumerative combinatorics, Volume 2, Cambridge Univ. Press, Cambridge, UK, 1999. 